\title{
Roles of FGFs As Paracrine or Endocrine Signals in Liver Development, Health, and Disease
}

\author{
Nobuyuki Itoh $^{1 *}$, Yoshiaki Nakayama ${ }^{2}$ and Morichika Konishi ${ }^{2}$ \\ ${ }^{1}$ Medical Innovation Center, Kyoto University Graduate School of Medicine, Kyoto, Japan, ${ }^{2}$ Department of Microbial \\ Chemistry, Kobe Pharmaceutical University, Kobe, Japan
}

The liver plays important roles in multiple processes including metabolism, the immune system, and detoxification and also has a unique capacity for regeneration. FGFs are growth factors that have diverse functions in development, health, and disease. The FGF family now comprises 22 members. Several FGFs have been shown to play roles as paracrine signals in liver development, health, and disease. FGF8 and FGF10 are involved in embryonic liver development, FGF7 and FGF9 in repair in response to liver injury, and FGF5, FGF8, FGF9, FGF17, and FGF18 in the development and progression of hepatocellular carcinoma. In contrast, FGF15/19 and FGF21 are endocrine signals.

OPEN ACCESS

Edited by:

Roland Wohlgemuth,

SIGMA-ALDRICH, Member of MERCK Group, Switzerland

Reviewed by: Massimo Broggini, Istituto di Ricerche Farmacologiche "Mario Negri", Italy Gaetano Romano,

Temple University, USA

*Correspondence:

Nobuyuki ltoh nobuyuki.itoh.56c@st.kyoto-u.ac.jp

Specialty section:

This article was submitted to

Molecular Medicine,

a section of the journal

Frontiers in Cell and Developmental

Biology

Received: 05 February 2016 Accepted: 27 March 2016

Published: 13 April 2016

Citation:

Itoh N, Nakayama Y and Konishi M (2016) Roles of FGFs As Paracrine or

Endocrine Signals in Liver Development, Health, and Disease.

Front. Cell Dev. Biol. 4:30.

doi: 10.3389/fcell.2016.00030
FGF15/19, which is produced in the ileum, is a negative regulator of bile acid metabolism and a stimulator of gallbladder filling. FGF15/19 is a postprandial, insulin-independent activator of hepatic protein and glycogen synthesis. It is also required for hepatocellular carcinoma and liver regeneration. FGF21 is a hepatokine produced in the liver. FGF21 regulates glucose and lipid metabolism in white adipose tissue. Serum FGF21 levels are elevated in non-alcoholic fatty liver. FGF21 also protects against non-alcoholic fatty liver. These findings provide new insights into the roles of FGFs in the liver and potential therapeutic strategies for hepatic disorders.

Keywords: carcinoma, development, disease, FGF, liver, metabolism

\section{INTRODUCTION}

The prototypic fibroblast growth factors (FGFs), FGF1 and FGF2, which were originally isolated from the brain as growth factors for fibroblasts, are multi-functional signaling proteins of $\sim 150$ amino acids that exert diverse activities in cell proliferation, angiogenesis, neuronal cell growth and survival, and wound healing with widespread expression profiles in embryos and adults (Burgess and Maciag, 1989; Baird and Klagsbrun, 1991). The FGF family now comprises 22 members including FGF1-FGF23 in humans and mice. These FGFs also have diverse functions in development, health, and disease. The human and mouse FGF families do not include FGF15 or FGF19, respectively, because they are orthologs. Although these orthologs have been named FGF15 in rodents and FGF19 in other vertebrates, they are typically referred to as FGF15/19 (Goetz and Mohammadi, 2013; Ornitz and Itoh, 2015).

The liver, which is the largest organ in the body, plays important roles in multiple processes including catabolism and anabolism, the immune system, and detoxification. The liver also has a unique capacity for regeneration with the potential for the full restoration of liver mass and function even following massive damage (Taub, 2004; Bhatia et al., 2014). Several FGFs function as paracrine or endocrine signals in liver development, health, and disease. These findings provide new insights 
into the roles of FGFs in the liver and potential therapeutic strategies for hepatic disorders. A succinct review of the roles of FGFs in the liver is provided herein.

\section{THE FGF FAMILY}

The FGF family includes 22 FGF proteins of $\sim 150-300$ amino acids with a conserved core $(\sim 30-60 \%$ amino acid identity) of $\sim 120$ amino acids. Phylogenetic analyses of the FGF family have revealed potential evolutionary relationships with seven subfamilies in this family. FGFs have been classified into paracrine, endocrine, and intracrine FGFs based on their mechanisms of action (Figure 1). Mice lacking these FGFs indicate their crucial roles in development and health. Paracrine FGFs, which comprise 15 members, are locally secreted signals that mainly function in multiple developmental and physiological processes. Endocrine FGFs, which comprise three members, are secreted endocrine signals that mainly function in multiple metabolic processes. FGF gene variations in humans also result in various diseases. These findings indicate that paracrine and endocrine FGFs are crucial for ensuring proper development and health in mice and humans. In contrast, intracrine FGFs, which comprise four members, are not secreted signals that play roles in the regulation of electrical excitability in neurons in an intracrine manner (Goetz and Mohammadi, 2013; Ornitz and Itoh, 2015). Several paracrine and endocrine FGFs, but not intracrine FGFs are involved in liver development, health, and disease as described below.

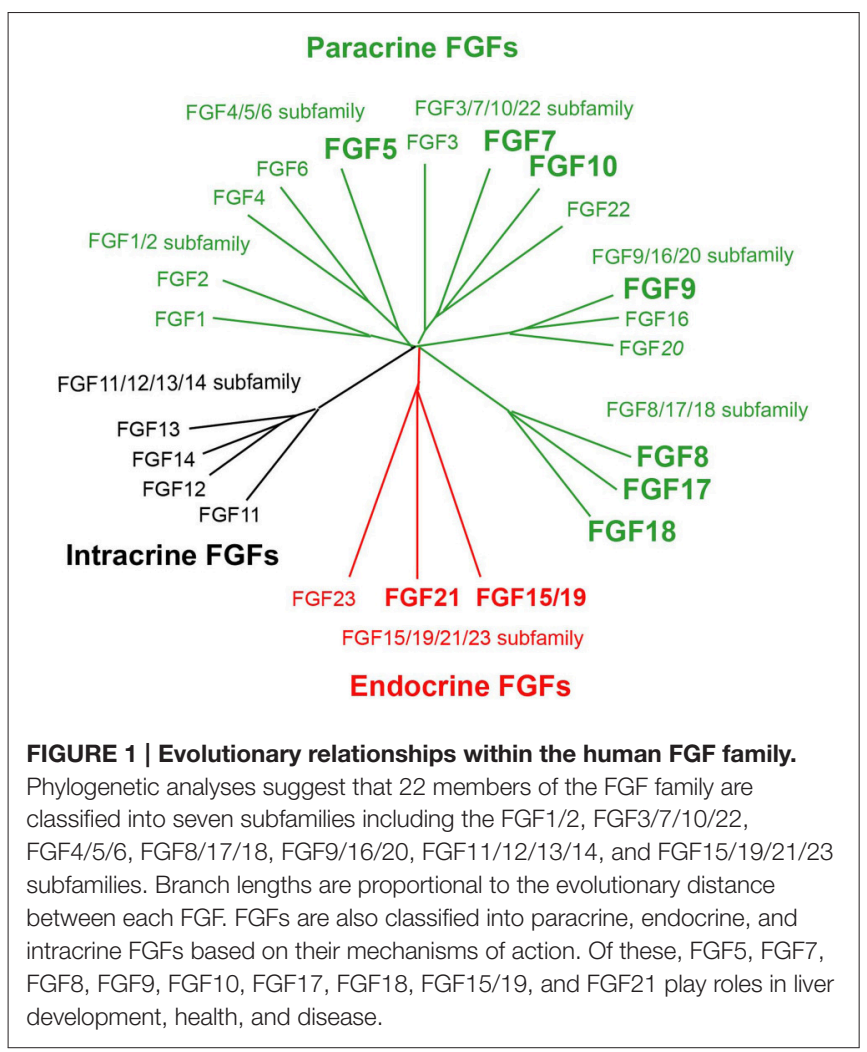

\section{ROLES OF PARACRINE FGFs IN THE LIVER}

Paracrine FGFs, which comprise 15 members including FGF1FGF6, FGF7-FGF10, FGF16-FGF18, FGF20, and FGF22, have a secreted signal sequence and heparan sulfate-binding site at their amino and carboxyl termini, respectively (Figures 1, 2A). Paracrine FGFs act on nearby target cells as locally secreted signals via diffusion. Heparan sulfate chains, which are long linear carbohydrate chains of repeating sulfated glucuronic acid linked to $\mathrm{N}$-acetylglucosamine disaccharides, are covalently linked to specific cell surface transmembrane-type proteins. Heparan sulfate functions to sequester FGFs and modulate their diffusion. This modulation of diffusion directs paracrine FGFs as local signals (Figures 2B, 3; Goetz and Mohammadi, 2013; Ornitz and Itoh, 2015).

Paracrine FGFs mediate biological responses by binding to cell surface FGF receptors (FGFRs) with heparan sulfate as a co-factor. Seven major FGFR proteins (FGFRs 1b, 1c, 2b, 2c, 3b, 3c, and 4) with differing ligand-binding specificities are generated from the FGFR1, FGFR2, FGFR3, and FGFR4 genes by alternative splicing. Heparan sulfate is necessary for stable interactions with FGFRs, and also independently interacts with FGFs and FGFRs. The FGFFGFR-heparan sulfate complex leads to FGFR dimerization, which directs the activation of FGFR intracellular tyrosine kinase domains, followed by that of key intracellular signaling pathways including the RAS-mitogen-activated protein kinase

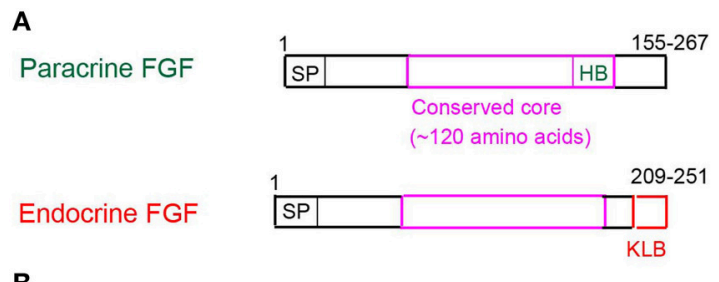

B

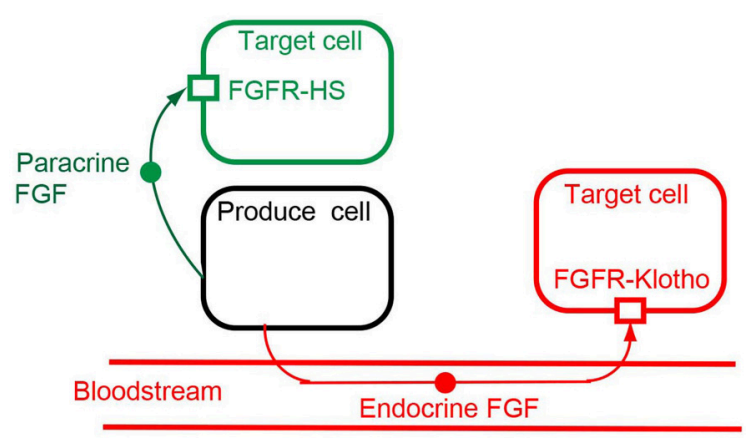

FIGURE 2 | (A) Schematic representations of paracrine and endocrine FGF structures. SP, HB, and KLB indicate a secreted signal sequence, heparan sulfate-binding site, and Klotho-binding site, respectively. (B) Mechanisms of action of paracrine and endocrine FGFs. Paracrine FGFs are locally secreted signals that act on nearby target cells by diffusion, with functions in multiple developmental and physiological processes. Endocrine FGFs are secreted endocrine signals that act on distant target cells through the bloodstream, with functions in multiple metabolic processes. FGFR-HS and FGFR-Klotho indicate the FGFR-heparan sulfate complex and FGFR-Klotho complex, respectively. 


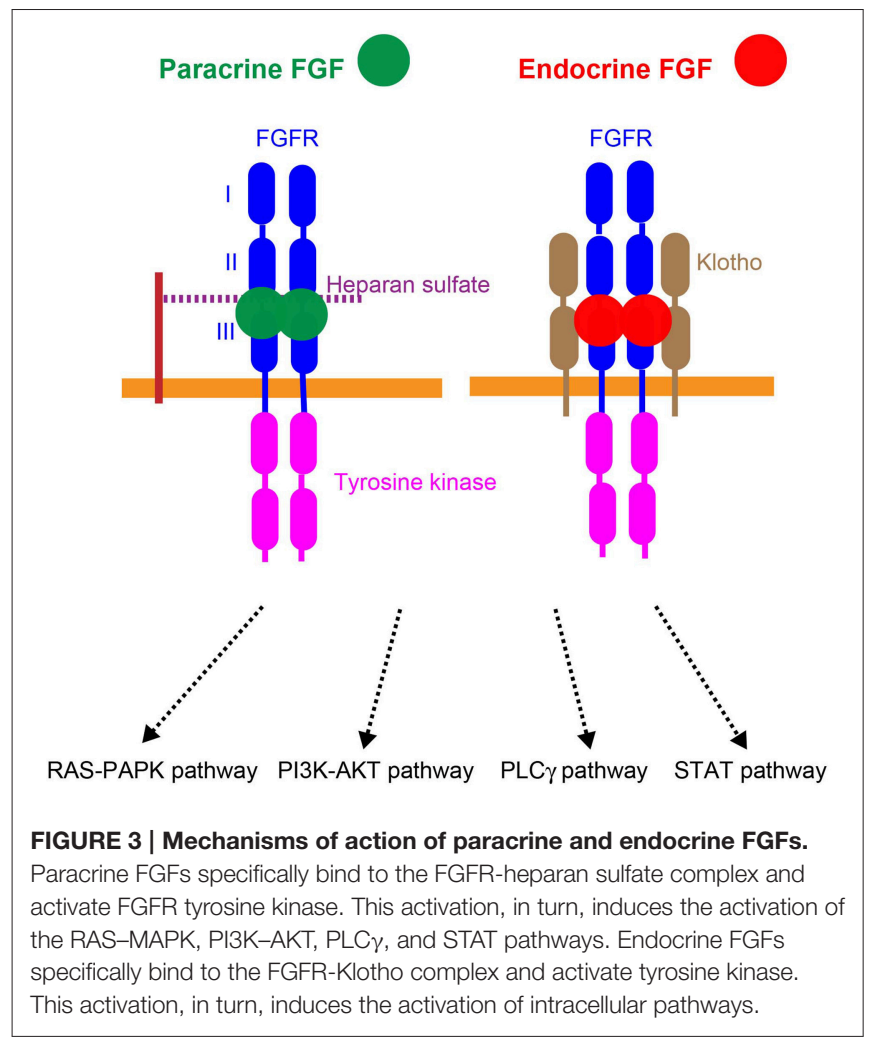

(MAPK), phosphoinositide 3-kinase (PI3K)-AKT, phospholipase $\mathrm{C} \gamma$ (PLC $\gamma)$, and signal transducer and activator of transcription (STAT) pathways (Figure 3; Goetz and Mohammadi, 2013; Ornitz and Itoh, 2015).

\section{Development}

The definitive endoderm, one of the embryonic germ layers, produces the gut tube and associated organs including the liver, lungs, and pancreas. Liver development, which is initiated by liver budding, occurs through reciprocal inductive interactions between the endoderm and underlying mesoderm. Secreted signals from the mesoderm to the endoderm are essential for liver budding. These secreted signals include FGFs, bone morphogenetic proteins (BMPs), and Wnts (Calmont et al., 2006; Tremblay, 2010). FGF8 and FGF10 play roles as paracrine signals in liver development (Table 1).

\section{FGF8 as a Morphogen}

FGF8 mainly activates FGFR1c with heparan sulfate as a cofactor. FGF8 is expressed in the adjacent mesoderm in the early embryonic stages, indicating its involvement in liver development (Crossley and Martin, 1995; Calmont et al., 2006; Wang et al., 2015). Since FGF8 knockout mice, which are lethal at the gastrulation stage, lack all embryonic mesoderm and endoderm-derived structures, the examination of its roles in liver development has been precluded (Sun et al., 1999). However, FGF8 is required for anterior heart field development; therefore, FGF8 is also expected to be required for liver development (Table 1; Ilagan et al., 2006).
TABLE 1 | Roles of paracrine/endocrine FGFs in the liver.

\begin{tabular}{|c|c|c|}
\hline FGFs & Roles & Species \\
\hline \multicolumn{3}{|c|}{ PARACRINE FGFs } \\
\hline FGF8 & A morphogen in liver development & Mice \\
\hline FGF10 & A morphogen in liver development & Mice \\
\hline FGF7 & A repair factor for liver injury & Mice/Humans \\
\hline FGF9 & A repair factor for liver injury & Mice \\
\hline FGF5 & A protector for NASH induced by a high-fat diet & Mice \\
\hline FGF8 & A progressor in $\mathrm{HCC}$ & Humans \\
\hline FGF17 & A progressor in $\mathrm{HCC}$ & Humans \\
\hline FGF18 & A progressor in $\mathrm{HCC}$ & Humans \\
\hline FGF5 & A target of miRNA in $\mathrm{HCC}$ & Humans \\
\hline FGF9 & A target of miRNA in $\mathrm{HCC}$ & Humans \\
\hline \multicolumn{3}{|c|}{ ENDOCRINE FGFs } \\
\hline FGF15/19 & $\begin{array}{l}\text { An ilium-derived regulator in hepatic bile acid, } \\
\text { protein, and, glycogen metabolism }\end{array}$ & Mice \\
\hline FGF21 & $\begin{array}{l}\text { A liver-derived regulator in glucose and lipid } \\
\text { metabolism }\end{array}$ & Mice \\
\hline FGF21 & A liver-derived protector for NAFLD/NASH & Mice/Humans \\
\hline FGF15/19 & An ilium-derived factor in liver regeneration & Mice \\
\hline FGF15/19 & An ilium-derived progressor in HCC & Mice/Humans \\
\hline
\end{tabular}

\section{FGF10 as a Morphogen}

FGF10 preferentially activates FGFR2b with heparan sulfate as a co-factor. FGF10 is also expressed in the adjacent mesoderm in the early embryonic stages, indicating its involvement in liver development (Kelly et al., 2001). FGF10 knockout mice are lethal shortly after birth due to the lack of multiple organs including limbs and lungs (Sekine et al., 1999). In addition, FGF10 knockout mice with smaller livers exhibit the reduced proliferation and survival of hepatoblasts, indicating that FGF10 is required for liver growth during embryogenesis and hepatoblast growth (Table 1; Berg et al., 2007).

\section{Repair}

The liver mainly comprises two types of cells: parenchymal (hepatocytes) and non-parenchymal cells. Hepatocytes, which account for $\sim 80 \%$ of hepatic cells, perform most metabolic functions in the liver. Hepatocytes also maintain the ability to proliferate in response to toxic injury and infection (Taub, 2004). FGF7 and FGF9 play roles as paracrine signals in liver repair (Table 1).

\section{FGF7 as a Repair Factor}

FGF7 preferentially activates FGFR2b with heparan sulfate as a co-factor. FGF7 is a paracrine FGF expressed in multiple tissues including the cerebrum, lungs, vas deferens, tongue, and skin in the postnatal stages (Fon Tacer et al., 2010). When hepatocyte proliferation is impaired by severe liver damage, facultative liver progenitor cells proliferate and contribute to repair. The expansion of liver progenitor cells is also often observed in patients with liver diseases. FGF7 knockout mice, which are viable, have impaired hair, kidney, and neuronal synapse development, but not impaired liver development (Guo et al., 1996; Qiao et al., 1999; Terauchi et al., 2010). However, 
liver progenitor cell expansion and higher mortality upon toxin-induced hepatic injury are markedly depressed in FGF7 knockout mice. In contrast, liver progenitor cells are induced and hepatic dysfunction is ameliorated in FGF7 transgenic mice. The expression of FGF7 is also induced concomitantly with liver progenitor cell responses in the livers of mouse models as well as in the serum of patients with acute liver failure. These findings indicate that FGF7 is a critical regulator of liver progenitor cells in liver injury (Table 1; Takase et al., 2013).

\section{FGF9 as a Repair Factor}

FGF9 mainly activates FGFR1c with heparan sulfate as a cofactor. FGF9 is broadly expressed at high levels throughout the brain and in the kidney in the postnatal stages (Fon Tacer et al., 2010). FGF9 knockout mice are lethal at the neonatal stage due to lung hypoplasia, but do not have impaired livers (Colvin et al., 2001). Hepatic injury is associated with the activation of hepatic stellate cells. The expression of FGF9 is increased in hepatic stellate cells in liver slice cultures after exposure to carbon tetrachloride as an acute liver injury model. FGF9 significantly stimulates the incorporation of thymidine by hepatocytes. These findings indicate that FGF9 provides a paracrine mitogenic signal to hepatocytes during acute liver injury (Table 1; Antoine et al., 2007).

\section{Non-alcoholic Fatty Liver Disease (NAFLD)}

NAFLD includes metabolic liver disorders ranging from simple fatty liver (hepatic steatosis) to non-alcoholic steatohepatitis (NASH) and liver cirrhosis. NAFLD, which has high morbidity and mortality rates, is regarded as a serious public health issue. The main risk factors for NAFLD are obesity, dyslipidemia, and insulin resistance. A multi-hit process including lipotoxicity, oxidative stress, endoplasmic reticulum stress, and an inflammatory state has been implicated in the pathogenesis of NAFLD (Takaki et al., 2013; Demir et al., 2015). FGF5 functions as a paracrine signal in NASH (Table 1).

\section{FGF5 Knockout Mice Fed a High-Fat Diet with the Characteristics of NASH}

FGF5 is expressed throughout the central nervous system and is also present in the skin in the postnatal stages (Fon Tacer et al., 2010). FGF5 knockout mice with the long hair phenotype are viable and appear to be healthy (Hébert et al., 1994). However, FGF5 knockout mice fed a high-fat diet gain little weight and have higher serum alanine transaminase, aspartate amino transferase, and non-high-density lipoprotein-cholesterol levels. Their liver histology indicates marked inflammation, focal necrosis, fat deposition, and fibrosis, which are similar to the characteristics of NASH. However, the mechanisms underlying the associations between FGF5, high-fat diet, and NASH currently remain unclear (Table 1; Hanaka et al., 2014).

\section{Hepatocellular Carcinoma (HCC)}

HCC is the most common type of liver cancer and has a poor prognosis. Most cases of HCC are secondary to a viral hepatitis infection or cirrhosis. Hepatocarcinogenesis in cirrhosis involves multiple processes, in which precancerous dysplastic nodules transform into early HCC, progressed HCC, and advanced HCC (Forner et al., 2012; Schulze et al., 2015). FGF signaling plays crucial roles in HCC. FGFR3 and FGFR4, which are the main FGFRs expressed in the liver, are involved in HCC (Qiu et al., 2005; French et al., 2012). FGFR2 has also been shown to play a role in HCC (Harimoto et al., 2010). In addition, FGF8, FGF17, and FGF18 act as paracrine signals in HCC. FGF5 and FGF9 are also targets of miRNAs in HCC (Table 1).

\section{FGF8, FGF17, and FGF18 as Progressors}

FGF8, FGF17, and FGF18 are members of the FGF8/7/18 subfamily (Figure 1). These FGFs mainly activate FGFR1c with heparan sulfate as a co-factor. At least one member of the FGF8/17/18 subfamily is up-regulated in 59\% of 34 human HCC cases. The expression of subfamily members is markedly increased in cultured HCC cells subjected to serum withdrawal or a hypoxia-mimetic drug. The addition of FGF8, FGF17, or FGF18 impairs apoptosis, the incidence of which is elevated in serumstarved cells. In contrast, the down-regulation of FGF18 by small interfering RNA significantly reduces the viability of HCC cells. FGF8, FGF17, and FGF18 are involved in autocrine/paracrine signaling in HCC and enhance the survival of tumor cells. These findings indicate that FGF8 subfamily members support the development and progression of hepatocellular malignancy (Table 1; Gauglhofer et al., 2011).

\section{FGF5 and FGF9 as Targets of microRNA (miRNAs)}

miRNAs are highly conserved small non-coding regulatory RNAs that negatively regulate gene expression by binding directly to corresponding target mRNAs in a sequence-specific manner (Giordano and Columbano, 2013). $m i R-188-5 p$ is significantly decreased in HCC cells and strongly correlates with multiple nodules, microvascular invasion, and the overall and diseasefree survival of HCC. The ectopic expression of miR-188-5p suppresses HCC cell proliferation and metastasis. The enforced expression of $m i R-188-5 p$ significantly inhibits the expression of FGF5, while the restoration of FGF5 expression reverses the inhibitory effects of miR-188-5p on HCC cell proliferation and metastasis. These findings indicate a tumor suppressor role for $m i R-188-5 p$ via the targeting of FGF5, which mainly activates FGFR1c with heparan sulfate as a co-factor in human HCC (Table 1; Fang et al., 2015). Furthermore, miR-140-5p suppresses tumor growth and metastasis by targeting FGF9, which also mainly activates FGFR1c with heparan sulfate as a co-factor, in human HCC (Table 1; Kuro-o, 2012; Yang et al., 2013).

\section{ROLES OF ENDOCRINE FGFs IN THE LIVER}

Endocrine FGFs, which comprise three members: FGF15/19, FGF21, and FGF23, have a secreted signal sequence and Klothobinding site at their amino and carboxyl termini (Figure 2A). In contrast to paracrine FGFs, endocrine FGFs do not function as local signals due to their lower heparan sulfate-binding affinity. 
They require $\alpha$ Klotho or $\beta$ Klotho as a co-factor for FGFR. $\alpha$ Klotho and $\beta$ Klotho are specifically expressed in the target tissues of endocrine FGFs, which function in an endocrine manner with target-tissue specificity through the bloodstream (Figure 2B; Goetz and Mohammadi, 2013; Ornitz and Itoh, 2015).

$\alpha$ Klotho and $\beta$ Klotho, which share structural similarities and characteristics with each other, are single-pass transmembrane proteins of $\sim 1000$ amino acids with a short cytoplasmic domain. However, endocrine FGFs cannot efficiently bind to FGFR, $\alpha$ Klotho, or $\beta$ Klotho alone; they efficiently bind to the FGFRKlotho complex. FGF15/19 activates FGFR4 with $\beta$ Klotho and FGF21 activates FGFR1c with $\beta$ Klotho, which, in turn, induces the activation of intracellular signaling pathways (Figure 3; Beenken and Mohammadi, 2012; Kuro-o, 2012). FGF15/19 exhibits metabolic and proliferative activities. However, FGF21 is a unique FGF with metabolic, but no proliferative activity (Goetz and Mohammadi, 2013; Ornitz and Itoh, 2015).

\section{Metabolism}

The liver plays important roles in multiple catabolic and anabolic pathways including bile acids, lipids, and carbohydrates, in addition to detoxification (Taub, 2004; Bhatia et al., 2014). FGF15/19 acts as an endocrine signal in bile acid metabolism in the liver. FGF21 also functions as an endocrine hepatokine in glucose and lipid metabolism (Table 1).

\section{FGF15/19 as a Regulator of Bile Acid Metabolism}

Most FGF15/19 knockout mice gradually die after embryonic day (E) 10.5 due to defects in the cardiac outflow tract, indicating that FGF15/19 plays a crucial role in embryonic heart development (McWhirter et al., 1997; Vincentz et al., 2005). However, few FGF15/19 knockout mice survive, even after the postnatal stages. In the postnatal stages, FGF15/19 is preferentially expressed in the ileum. Bile acids, which are synthesized in the liver, promote the digestion and absorption of dietary fat by forming micelles. The liver and intestines play crucial roles in maintaining bile acid homeostasis. Cholesterol $7 \alpha$-hydroxylase (CYP7A1) catalyzes the first and rate-limiting step in the bile acid synthetic pathway in the liver. The expression of FGF15/19 in the ileum is induced by the nuclear bile acid receptor FXR. The induction of FGF15/19 represses CYP7A1 expression in the liver in an endocrine manner. Surviving FGF15/19 knockout mice have higher CYP7A1 expression levels and enhanced fecal bile acid excretion, indicating that FGF15/19 plays a crucial role in bile acid synthesis as a gut-liver signal in an endocrine manner (Table 1; Inagaki et al., 2005). The cycle of gallbladder filling and emptying controls the flow of bile into the intestines for digestion. Gallbladders in surviving FGF15/19 knockout mice are almost devoid of bile. The gallbladder volume in surviving FGF15/19 knockout mice is significantly increased by an injection of the FGF15/19 protein. These findings indicate that FGF15/19 also plays crucial roles in gallbladder filling (Table 1; Choi et al., 2006). FGF15/19 stimulates hepatic protein and glycogen synthesis, but does not induce lipogenesis. Surviving FGF15/19 knockout mice fail to properly maintain blood concentrations of glucose and normal postprandial amounts of liver glycogen. FGF15/19 treatments restore the loss of glycogen in streptozotocin-treated mice that are diabetic and have no detectable blood insulin levels. These findings indicate that FGF15/19 is a postprandial, insulinindependent activator that acts directly on the liver via hepatic protein and glycogen synthesis (Table 1; Kir et al., 2011).

\section{FGF21 as a Hepatokine in Glucose and Lipid Metabolism}

Liver-derived cytokines are known to function as hepatokines and include angiopoietin-related protein 6, fetuin-A, insulinlike growth factors, and selenoprotein P. Hepatokines directly regulate glucose and lipid metabolism (Stefan and Häring, 2013). FGF21, which is abundantly expressed in the liver, also plays roles as a hepatokine in glucose and lipid metabolism in white adipose tissue (Itoh, 2014). Although hepatic FGF21 is generally expressed at low levels, its expression is strongly induced during fasting through the activation of peroxisome proliferator-activated receptor $\alpha(\operatorname{PPAR} \alpha)$ by the non-esterified fatty acids released from adipocytes and taken up by hepatocytes (Murata et al., 2011). FGF21 knockout mice are viable, fertile, and appear to be normal. Lipolysis in white adipose tissue is enhanced in fasted Fgf21 knockout mice, indicating that FGF21 inhibits lipolysis during fasting (Hotta et al., 2009). Hepatic Fgf21 expression is also significantly induced by a low-carbohydrate, high-fat ketogenic diet. Insulin sensitivity in white adipose tissue is impaired by ketogenic diet feeding. This impaired sensitivity is improved in FGF21 knockout mice, indicating that FGF21 is a negative regulator of adipocyte insulin sensitivity in adaptation to a low-carbohydrate malnutritional state (Murata et al., 2013). Hepatic FGF21 induced by starvation also increases systemic glucocorticoid levels and suppresses physical activity in adaptation to starvation responses. These effects in mice were not observed in perfused livers or cultured hepatocytes (Ogawa et al., 2007; Potthoff et al., 2009). In addition, most of these effects have not been observed in mice lacking $\beta$-Klotho in the brain or those lacking FGFR1 in adipose tissue (Adams et al., 2010; Owen et al., 2014). These findings indicate that hepatic FGF21 exerts diverse actions through FGFR1c/ $\beta$-Klotho in the brain or adipose tissue, but not directly on the liver. Hepatic FGF21 expression is also significantly induced by different kinds of stresses such as hepatic injury, chemical insults, and diseases, indicating that hepatic FGF21 is a stress-induced metabolic regulator (Table 1; Cheng et al., 2014).

Adiponectin is a white adipocyte-derived hormone. Studies on FGF21 knockout mice have also indicated that hepatic FGF21 is an upstream effector of adiponectin that mediates many of the systemic effects of FGF21 on energy metabolism and insulin sensitivity in the liver and skeletal muscle (Holland et al., 2013; Lin et al., 2013). Glucagon regulates glucose and lipid metabolism and promotes weight loss. Glucagon receptor activation increases hepatic FGF21 expression. Findings obtained from FGF21 knockout mice suggest that hepatic FGF21 contributes, at least in part, to glucose, energy, and lipid metabolism controlled by glucagon (Habegger et al., 2013). FGF21 knockout mice also exhibit insulin resistance while being normoglycemic, and this 
is associated with increases in pancreatic beta-cell proliferation and insulin synthesis, which act as compensatory responses. This resistance results from enhanced growth hormone sensitivity in FGF21 knockout pancreatic islets, indicating that hepatic FGF21 is important for the regulation of pancreatic beta-cell proliferation and insulin synthesis, possibly via the modulation of growth hormone signaling (So et al., 2015). Endoplasmic reticulum (ER) stress leads to the development and progression of various diseases such as obesity and diabetes. ER stress induces hepatic FGF21 expression. ER stress and the hepatic accumulation of lipids are enhanced in FGF21 knockout mice, indicating that FGF21 plays a role in adaptive responses to ER stress (Table 1; Kim et al., 2015).

\section{NAFLD}

As described above, NAFLD includes metabolic liver disorders ranging from hepatic steatosis to NASH and liver cirrhosis. FGF21 plays roles in NAFLD (Table 1).

\section{FGF21 as a Protective Factor for NAFLD}

Serum FGF21 levels are significantly increased in patients with hepatic steatosis and NASH in a manner that is dependent on the degree of steatosis (Yilmaz and Eren, 2012; Shen et al., 2012, 2013; Li et al., 2013; Liu et al., 2015). Mice fed a high-fat diet or methionine/choline-deficient diet are model mice for NASH. Serum FGF21 levels and hepatic FGF21 expression levels are also significantly higher in these mice (Xu et al., 2009; Tanaka et al., 2015). As described above, FGF21 is an important metabolic regulator of glucose and lipid metabolism. The administration of a high dose of intravenous FGF21 reverses hepatic steatosis, improves insulin sensitivity, and decreases serum glucose levels in mice with NAFLD (Xu et al., 2009). These findings indicate that elevated serum FGF21 levels may be a protective response against glucose-lipid metabolism disorders in patients and mice with NAFLD/NASH (Table 1).

Metformin decreases hepatic gluconeogenesis and increases hepatic fatty acid $\beta$-oxidation by activating AMPK (Doycheva and Loomba, 2014). In cultured hepatocytes, metformin stimulates the expression of FGF21, which is inhibited by an AMPK inhibitor, indicating that FGF21 may contribute to the therapeutic effects of metformin on NAFLD (Nygaard et al., 2012). However, metformin is not beneficial for patients with NAFLD (Mazza et al., 2012). The nicotinamide adenine dinucleotide-dependent deacetylase sirtuin 1 (SIRT1) tightly regulates fatty acid metabolism in the liver. Prolonged fasting induces lipid deposition in the livers of wild-type mice, but severe hepatic steatosis in liver-specific SIRT1 knockout mice. Fasting increases the expression of FGF21 in the livers of wildtype mice, but not in those of liver-specific SIRT1 knockout mice. Decreased hepatic FGF21 expression and serum FGF21 levels in fasted liver-specific SIRT1 knockout mice have been correlated with the decreased hepatic expression of genes involved in fatty acid oxidation and ketogenesis, and increased expression of genes that control lipogenesis. The SIRT1 activator, resveratrol, increases FGF21 mRNA and FGF21 protein levels in human liver carcinoma HepG2 cells. The hepatic overexpression of
FGF21 in liver-specific SIRT1 knockout mice increases the expression of genes involved in fatty acid oxidation, thereby decreasing fasting-induced steatosis, reducing obesity, increasing energy expenditure, and promoting the browning of white adipose tissue. These findings indicate that the SIRT1-mediated activation of FGF21 prevents liver steatosis caused by fasting (Table 1; Li et al., 2014).

\section{Regeneration}

Hepatocytes, which are long lived, normally do not undergo cell division. After the surgical removal of two-thirds of the liver in mice, the remaining liver enlarges until the original liver is restored. The liver has a strong regenerative capacity due to hyperplastic responses that involve the replication of all mature functioning cells in the remnant liver without the recruitment of liver stem cells or progenitor cells (Taub, 2004). FGF15/19 functions as an endocrine signal in liver regeneration (Table 1).

\section{FGF15/19 as a Regenerative Factor}

As described above, FGF15/19 is preferentially expressed in the ileum. After 2/3 partial hepatectomy, FGF15/19 knockout mice display more extensive liver necrosis and greater elevations in serum bile acid and bilirubin levels than wild-type mice. In addition, hepatocyte proliferation is reduced in FGF15/19 knockout mice because of impaired cell cycle progression, indicating that FGF15/19 is required for liver regeneration. The underlying mechanisms are likely the result of disrupted bile acid homeostasis and the impaired priming of hepatocyte proliferation (Table 1; Uriarte et al., 2013; Kong et al., 2014).

\section{HCC}

As described above, HCC is the most common type of liver cancer, in which FGF15/19 plays roles (Table 1).

\section{FGF15/19 as a Progressor}

Fewer and smaller tumors with smaller histological neoplastic lesions are observed in FGF15/19 knockout mice subjected to a clinically relevant model of liver inflammation and fibrosis-associated carcinogenesis. Ileal FGF15/19 expression is stimulated in mice undergoing carcinogenesis. Hepatocellular proliferation and fibrogenesis are also reduced in FGF15/19 knockout mice. In vitro experiments indicate that liver fibrogenic stellate cells are not direct targets for FGF15/FGF19. FGF15/FGF19 as an endocrine factor induces the expression of pro-fibrogenic and pro-tumorigenic connective tissue growth factor (CTGF) in hepatocytes. These findings indicate the existence of FGF15/19-triggered CTGF-mediated paracrine effects on stellate cells, and an amplification mechanism for the hepatocarcinogenic effects of FGF15/19 via the production of CTGF (Table 1; Uriarte et al., 2015).

In contrast, FGF15/19 is significantly overexpressed in human HCC. Serum FGF15/19 levels in patients with HCC are significantly decreased after hepatectomy. The FGF15/19 
protein increases the proliferation and inversion capabilities of cultured human hepatocellular carcinoma cells. These findings indicate that FGF15/19 functions as an autocrine/paracrine factor in human HCC (Miura et al., 2012). Epithelial-mesenchymal transition is a key event in metastasis and plays a critical role in the progression of HCC (van Zijl et al., 2009). The expression of FGF15/19 is significantly elevated and negatively associated with the expression of E-cadherin in HCC tissues and cell lines. Ectopic FGF15/19 expression promotes epithelialmesenchymal transition and invasion in epithelial-like HCC cells through the repression of E-cadherin expression, whereas FGF15/19 knockdown enhances E-cadherin expression and, hence, diminishes epithelial-mesenchymal transition traits in mesenchymal-like HCC cells. However, FGF15/19 knockdown cannot abrogate epithelial-mesenchymal transition traits in the presence of glycogen synthase kinase $3 \beta$ (GSK3 $\beta$ ) inhibitors. FGF15/19-induced epithelial-mesenchymal transition may be markedly attenuated when FGFR4 is knocked out. These findings indicate that the FGFR4/GSK3 $\beta / \beta$-catenin axis plays a pivotal role in FGF15/19-induced epithelial-mesenchymal transition in HCC cells (Table 1; Zhao et al., 2015).

Genomic analyses promise to improve tumor characterization in order to optimize personalized treatments for patients with HCC. Exome sequencing analyses of HCC indicate that the gene locus including FGF15/19 is amplified at advanced stages in aggressive HCC (Table 1; Schulze et al., 2015).

\section{REFERENCES}

Adams, A. C., Yang, C., Coskun, T., Cheng, C. C., Gimeno, R. E., Luo, Y., et al. (2010). The breadth of FGF21's metabolic actions are governed by FGFR1 in adipose tissue. Mol. Metab. 2, 31-37. doi: 10.1016/j.molmet.2012. 08.007

Antoine, M., Wirz, W., Tag, C. G., Gressner, A. M., Marvituna, M., Wycislo, M., et al. (2007). Expression and function of fibroblast growth factor (FGF) 9 in hepatic stellate cells and its role in toxic liver injury. Biochem. Biophys. Res. Commun. 361, 335-341. doi: 10.1016/j.bbrc.2007.06.189

Baird, A., and Klagsbrun, M. (1991). The fibroblast growth factor family. Cancer Cells 3, 239-243.

Beenken, A., and Mohammadi, M. (2012). The structural biology of the FGF19 subfamily. Adv. Exp. Med. Biol. 728, 1-24. doi: 10.1007/978-1-4614-0887-1_1

Berg, T., Rountree, C. B., Lee, L., Estrada, J., Sala, F. G., Choe, A., et al. (2007). Fibroblast growth factor 10 is critical for liver growth during embryogenesis and controls hepatoblast survival via beta-catenin activation. Hepatology 46, 1187-1197. doi: 10.1002/hep.21814

Bhatia, S. N., Underhill, G. H., Zaret, K. S., and Fox, I. J. (2014). Cell and tissue engineering for liver disease. Sci. Transl. Med. 6, 245sr2. doi: 10.1126/scitranslmed.3005975

Burgess, W. H., and Maciag, T. (1989). The heparin-binding (fibroblast) growth factor family of proteins. Annu. Rev. Biochem. 58, 575-606. doi: 10.1146/annurev.bi.58.070189.003043

Calmont, A., Wandzioch, E., Tremblay, K. D., Minowada, G., Kaestner, K. H., Martin, G. R., et al. (2006). An FGF response pathway that mediates hepatic gene induction in embryonic endoderm cells. Dev. Cell 11, 339-348. doi: 10.1016/j.devcel.2006.06.015

Cheng, X., Vispute, S. G., Liu, J., Cheng, C., Kharitonenkov, A., and Klaassen, C. D. (2014). Fibroblast growth factor (Fgf) 21 is a novel target gene of the aryl hydrocarbon receptor (AhR). Toxicol. Appl. Pharmacol. 278, 65-71. doi: 10.1016/j.taap.2014.04.013

\section{CONCLUSIONS}

FGFs are growth factors with diverse functions in development, health, and disease. Among 22 FGFs, several FGFs act as paracrine or endocrine signals in liver development, health, and disease. FGF8 and FGF10 function as paracrine signals in embryonic liver development. FGF7 and FGF9 also act as paracrine signals in repair in response to liver injury. In addition, FGF5, FGF8, FGF9, FGF17, and FGF18 play roles as paracrine signals in the development and progression of hepatocellular carcinoma. In contrast, FGF15/19 and FGF21 are endocrine signals. FGF15/19, which is produced in the ileum, is involved in bile acid metabolism and gallbladder filling in the liver. FGF15/19 is also a postprandial, insulin-independent activator of hepatic protein and glycogen synthesis. Furthermore, FGF15/19 is involved in liver regeneration and hepatocellular carcinoma. FGF21 is a hepatokine produced in the liver. It regulates glucose and lipid metabolism. Serum FGF21 levels are increased in NAFLD. FGF21 also protects against NAFLD. These findings provide new insights into the roles of FGFs in the liver and potential therapeutic strategies for hepatic disorders.

\section{AUTHOR CONTRIBUTIONS}

All authors listed, have made substantial, direct and intellectual contribution to the work, and approved it for publication.

Choi, M., Moschetta, A., Bookout, A. L., Peng, L., Umetani, M., Holmstrom, S. R., et al. (2006). Identification of a hormonal basis for gallbladder filling. Nat. Med. 12, 1253-12555. doi: 10.1038/nm1501

Colvin, J. S., White, A. C., Pratt, S. J., and Ornitz, D. M. (2001). Lung hypoplasia and neonatal death in Fgf9-null mice identify this gene as an essential regulator of lung mesenchyme. Development 128, 2095-2106.

Crossley, P. H., and Martin, G. R. (1995). The mouse Fgf8 gene encodes a family of polypeptides and is expressed in regions that direct outgrowth and patterning in the developing embryo. Development $121,439-451$.

Demir, M., Lang, S., and Steffen, H. M. (2015). Nonalcoholic fatty liver disease: current status and future directions. J. Dig. Dis. 16, 541-557. doi: 10.1111/17512980.12291

Doycheva, I., and Loomba, R. (2014). Effect of metformin on ballooning degeneration in nonalcoholic steatohepatitis (NASH): when to use metformin in nonalcoholic fatty liver disease (NAFLD). Adv. Ther. 31, 30-43. doi: 10.1007/s12325-013-0084-6

Fang, F., Chang, R. M., Yu, L., Lei, X., Xiao, S., Yang, H., et al. (2015). MicroRNA-188-5p suppresses tumor cell proliferation and metastasis by directly targeting FGF5 in hepatocellular carcinoma. J. Hepatol. 63, 874-885. doi: 10.1016/j.jhep.2015.05.008

Fon Tacer, K., Bookout, A. L., Ding, X., Kurosu, H., John, G. B., Wang, L., et al. (2010). Research resource: comprehensive expression atlas of the fibroblast growth factor system in adult mouse. Mol. Endocrinol. 24, 2050-2064. doi: $10.1210 /$ me.2010-0142

Forner, A., Llovet, J. M., and Bruix, J. (2012). Chemoembolization for intermediate HCC: is there proof of survival benefit? J. Hepatol. 56, 984-986. doi: 10.1016/j.jhep.2011.08.017

French, D. M., Lin, B. C., Wang, M., Adams, C., Shek, T., Hötzel, K., et al. (2012). Targeting FGFR4 inhibits hepatocellular carcinoma in preclinical mouse models. PLoS ONE 7:e36713. doi: 10.1371/journal.pone.0036713

Gauglhofer, C., Sagmeister, S., Schrottmaier, W., Fischer, C., Rodgarkia-Dara, C., Mohr, T., et al. (2011). Up-regulation of the fibroblast growth factor 8 subfamily 
in human hepatocellular carcinoma for cell survival and neoangiogenesis. Hepatology 53, 854-864. doi: 10.1002/hep.24099

Giordano, S., and Columbano, A. (2013). MicroRNAs: new tools for diagnosis, prognosis, and therapy in hepatocellular carcinoma? Hepatology 57, 840-847. doi: $10.1002 /$ hep. 26095

Goetz, R., and Mohammadi, M. (2013). Exploring mechanisms of FGF signalling through the lens of structural biology. Nat. Rev. Mol. Cell. Biol. 14, 166-180. doi: $10.1038 / \mathrm{nrm} 3528$

Guo, L., Degenstein, L., and Fuchs, E. (1996). Keratinocyte growth factor is required for hair development but not for wound healing. Genes Dev. 10, 165-175. doi: 10.1101/gad.10.2.165

Habegger, K. M., Stemmer, K., Cheng, C., Müller, T. D., Heppner, K. M., Ottaway, N., et al. (2013). Fibroblast growth factor 21 mediates specific glucagon actions. Diabetes 62, 1453-1463. doi: 10.2337/db12-1116

Hanaka, H., Hamada, T., Ito, M., Nakashima, H., Tomita, K., Seki, S., et al. (2014). Fibroblast growth factor-5 participates in the progression of hepatic fibrosis. Exp. Anim. 63, 85-92. doi: 10.1538/expanim. 63.85

Harimoto, N., Taguchi, K., Shirabe, K., Adachi, E., Sakaguchi, Y., Toh, Y., et al. (2010). The significance of fibroblast growth factor receptor 2 expression in differentiation of hepatocellular carcinoma. Oncology 78, 361-368. doi: $10.1159 / 000320463$

Hébert, J. M., Rosenquist, T., Götz, J., and Martin, G. R. (1994). FGF5 as a regulator of the hair growth cycle: evidence from targeted and spontaneous mutations. Cell 78, 1017-1025. doi: 10.1016/0092-8674(94) 90276-3

Holland, W. L., Adams, A. C., Brozinick, J. T., Bui, H. H., Miyauchi, Y., Kusminski, C. M., et al. (2013). An FGF21-adiponectin-ceramide axis controls energy expenditure and insulin action in mice. Cell Metab. 17, 790-797. doi: 10.1016/j.cmet.2013.03.019

Hotta, Y., Nakamura, H., Konishi, M., Murata, Y., Takagi, H., Matsumura, S., et al. (2009). Fibroblast growth factor 21 regulates lipolysis in white adipose tissue but is not required for ketogenesis and triglyceride clearance in liver. Endocrinology 150, 4625-4633. doi: 10.1210/en. 2009-0119

Ilagan, R., Abu-Issa, R., Brown, D., Yang, Y. P., Jiao, K., Schwartz, R. J., et al. (2006). Fgf8 is required for anterior heart field development. Development 133, 2435-2445. doi: $10.1242 / \mathrm{dev} .02408$

Inagaki, T., Choi, M., Moschetta, A., Peng, L., Cummins, C. L., McDonald, J. G., et al. (2005). Fibroblast growth factor 15 functions as an enterohepatic signal to regulate bile acid homeostasis. Cell Metab. 2, 217-225. doi: 10.1016/j.cmet.2005.09.001

Itoh, N. (2014). FGF21 as a hepatokine, adipokine, and myokine in metabolism and diseases. Front. Endocrinol. 5:107. doi: 10.3389/fendo.2014.00107

Kelly, R. G., Brown, N. A., and Buckingham, M. E. (2001). The arterial pole of the mouse heart forms from Fgf10-expressing cells in pharyngeal mesoderm. Dev. Cell 1, 435-440. doi: 10.1016/S1534-5807(01)00040-5

Kim, S. H., Kim, K. H., Kim, H. K., Kim, M. J., Back, S. H., Konishi, M., et al. (2015). Fibroblast growth factor 21 participates in adaptation to endoplasmic reticulum stress and attenuates obesity-induced hepatic metabolic stress. Diabetologia 58 , 809-818. doi: 10.1007/s00125-014-3475-6

Kir, S., Beddow, S. A., Samuel, V. T., Miller, P., Previs, S. F., Suino-Powell, K., et al. (2011). FGF19 as a postprandial, insulin-independent activator of hepatic protein and glycogen synthesis. Science 331, 1621-1624. doi: 10.1126/science. 1198363

Kong, B., Huang, J., Zhu, Y., Li, G., Williams, J., Shen, S., et al. (2014). Fibroblast growth factor 15 deficiency impairs liver regeneration in mice. Am. J. Physiol. Gastrointest. Liver Physiol. 306, G893-G902. doi: 10.1152/ajpgi.00337.2013

Kuro-o, M. (2012). Klotho and BKlotho. Adv. Exp. Med. Biol. 728, 25-40. doi: 10.1007/978-1-4614-0887-1_2

Li, H., Dong, K., Fang, Q., Hou, X., Zhou, M., Bao, Y., et al. (2013). High serum level of fibroblast growth factor 21 is an independent predictor of non-alcoholic fatty liver disease: a 3-year prospective study in China. J. Hepatol. 58, 557-563. doi: $10.1016 /$ j.jhep.2012.10.029

Li, Y., Wong, K., Giles, A., Jiang, J., Lee, J. W., Adams, A. C., et al. (2014). Hepatic SIRT1 attenuates hepatic steatosis and controls energy balance in mice by inducing fibroblast growth factor 21. Gastroenterology 146, 539.e7-549.e7. doi: 10.1053/j.gastro.2013.10.059
Lin, Z., Tian, H., Lam, K. S., Lin, S., Hoo, R. C., Konishi, M., et al. (2013). Adiponectin mediates the metabolic effects of FGF21 on glucose homeostasis and insulin sensitivity in mice. Cell Metab. 17, 779-789. doi: 10.1016/j.cmet.2013.04.005

Liu, J., Xu, Y., Hu, Y., and Wang, G. (2015). The role of fibroblast growth factor 21 in the pathogenesis of non-alcoholic fatty liver disease and implications for therapy. Metabolism 64, 380-390. doi: 10.1016/j.metabol.2014.11.009

Mazza, A., Fruci, B., Garinis, G. A., Giuliano, S., Malaguarnera, R., and Belfiore, A. (2012). The role of metformin in the management of NAFLD. Exp. Diabetes Res. 2012:716404. doi: 10.1155/2012/716404

McWhirter, J. R., Goulding, M., Weiner, J. A., Chun, J., and Murre, C. (1997). A novel fibroblast growth factor gene expressed in the developing nervous system is a downstream target of the chimeric homeodomain oncoprotein E2A-Pbx1. Development 124, 3221-3232.

Miura, S., Mitsuhashi, N., Shimizu, H., Kimura, F., Yoshidome, H., Otsuka, M., et al. (2012). Fibroblast growth factor 19 expression correlates with tumor progression and poorer prognosis of hepatocellular carcinoma. BMC Cancer 12:56. doi: 10.1186/1471-2407-12-56

Murata, Y., Konishi, M., and Itoh, N. (2011). FGF21 as an endocrine regulator in lipid metabolism: from molecular evolution to physiology and pathophysiology. J. Nutr. Metab. 2011:981315. doi: 10.1155/2011/981315

Murata, Y., Nishio, K., Mochiyama, T., Konishi, M., Shimada, M., Ohta, H., et al. (2013). Fgf21 impairs adipocyte insulin sensitivity in mice fed a low-carbohydrate, high-fat ketogenic diet. PLOS ONE 8:e69330. doi: 10.1371/journal.pone. 0069330

Nygaard, E. B., Vienberg, S. G., Ørskov, C., Hansen, H. S., and Andersen, B. (2012). Metformin stimulates FGF21 expression in primary hepatocytes. Exp. Diabetes Res. 2012:465282. doi: 10.1155/2012/465282

Ogawa, Y., Kurosu, H., Yamamoto, M., Nandi, A., Rosenblatt, K. P., Goetz, R., et al. (2007). BetaKlotho is required for metabolic activity of fibroblast growth factor 21. Proc. Natl. Acad. Sci. U.S.A. 104, 7432-7437. doi: 10.1073/pnas.0701600104 Ornitz, D. M., and Itoh, N. (2015). The fibroblast growth factor signaling pathway. Wiley Interdiscip. Rev. Dev. Biol. 4, 215-266. doi: 10.1002/wdev.176

Owen, B. M., Ding, X., Morgan, D. A., Coate, K. C., Bookout, A. L., Rahmouni, K., et al. (2014). FGF21 acts centrally to induce sympathetic nerve activity, energy expenditure, and weight loss. Cell Metab. 20, 670-677. doi: 10.1016/j.cmet.2014.07.012

Potthoff, M. J., Inagaki, T., Satapati, S., Ding, X., He, T., Goetz, R., et al. (2009). FGF21 induces PGC-1alpha and regulates carbohydrate and fatty acid metabolism during the adaptive starvation response. Proc. Natl. Acad. Sci. U.S.A. 106, 10853-10858. doi: 10.1073/pnas.0904187106

Qiao, J., Uzzo, R., Obara-Ishihara, T., Degenstein, L., Fuchs, E., and Herzlinger, D. (1999). FGF-7 modulates ureteric bud growth and nephron number in the developing kidney. Development 126, 547-554.

Qiu, W. H., Zhou, B. S., Chu, P. G., Chen, W. G., Chung, C., Shih, J., et al. (2005). Over-expression of fibroblast growth factor receptor 3 in human hepatocellular carcinoma. World J. Gastroenterol. 11, 5266-5272. doi: 10.3748/wjg.v11.i34.5266

Schulze, K., Imbeaud, S., Letouzé, E., Alexandrov, L. B., Calderaro, J., Rebouissou, S., et al. (2015). Exome sequencing of hepatocellular carcinomas identifies new mutational signatures and potential therapeutic targets. Nat. Genet. 47, 505-511. doi: 10.1038/ng.3252

Sekine, K., Ohuchi, H., Fujiwara, M., Yamasaki, M., Yoshizawa, T., Sato, T., et al. (1999). Fgf10 is essential for limb and lung formation. Nat. Genet. 21, 138-141.

Shen, J., Chan, H. L., Wong, G. L., Choi, P. C., Chan, A. W., Chan, H. Y., et al. (2012). Non-invasive diagnosis of non-alcoholic steatohepatitis by combined serum biomarkers. J. Hepatol. 56, 1363-1370. doi: 10.1016/j.jhep.2011.12.025

Shen, Y., Ma, X., Zhou, J., Pan, X., Hao, Y., Zhou, M., et al. (2013). Additive relationship between serum fibroblast growth factor 21 level and coronary artery disease. Cardiovasc. Diabetol. 12:124. doi: 10.1186/1475-2840-12-124

So, W. Y., Cheng, Q., Xu, A., Lam, K. S., and Leung, P. S. (2015). Loss of fibroblast growth factor 21 action induces insulin resistance, pancreatic islet hyperplasia and dysfunction in mice. Cell Death Dis. 6, e1707. doi: 10.1038/cddis.2015.80

Stefan, N., and Häring, H. U. (2013). The role of hepatokines in metabolism. Nat. Rev. Endocrinol. 9, 144-152. doi: 10.1038/nrendo.2012.258

Sun, X., Meyers, E. N., Lewandoski, M., and Martin, G. R. (1999). Targeted disruption of Fgf8 causes failure of cell migration in the gastrulating mouse embryo. Genes Dev. 13, 1834-1846. doi: 10.1101/gad.13.14.1834 
Takaki, A., Kawai, D., and Yamamoto, K. (2013). Multiple hits, including oxidative stress, as pathogenesis and treatment target in non-alcoholic steatohepatitis (NASH). Int. J. Mol. Sci. 14, 20704-20728. doi: 10.3390/ijms141020704

Takase, H. M., Itoh, T., Ino, S., Wang, T., Koji, T., Akira, S., et al. (2013). FGF7 is a functional niche signal required for stimulation of adult liver progenitor cells that support liver regeneration. Genes Dev. 27, 169-181. doi: 10.1101/gad.204776.112

Tanaka, N., Takahashi, S., Zhang, Y., Krausz, K. W., Smith, P. B., Patterson, A. D., et al. (2015). Role of fibroblast growth factor 21 in the early stage of NASH induced by methionine- and choline-deficient diet. Biochim. Biophys. Acta 1852, 1242-1252. doi: 10.1016/j.bbadis.2015.02.012

Taub, R. (2004). Liver regeneration: from myth to mechanism. Nat. Rev. Mol. Cell Biol. 5, 836-847. doi: 10.1038/nrm 1489

Terauchi, A., Johnson-Venkatesh, E. M., Toth, A. B., Javed, D., Sutton, M. A., and Umemori, H. (2010). Distinct FGFs promote differentiation of excitatory and inhibitory synapses. Nature 465, 783-787. doi: 10.1038/nature 09041

Tremblay, K. D. (2010). Formation of the murine endoderm: lessons from the mouse, frog, fish, and chick. Prog. Mol. Biol. Transl. Sci. 96, 1-34. doi: 10.1016/B978-0-12-381280-3.00001-4

Uriarte, I., Fernandez-Barrena, M. G., Monte, M. J., Latasa, M. U., Chang, H. C., Carotti, S., et al. (2013). Identification of fibroblast growth factor 15 as a novel mediator of liver regeneration and its application in the prevention of post-resection liver failure in mice. Gut 62, 899-910. doi: 10.1136/gutjnl-2012302945

Uriarte, I., Latasa, M. U., Carotti, S., Fernandez-Barrena, M. G., Garcia-Irigoyen, O., Elizalde, M., et al. (2015). Ileal FGF15 contributes to fibrosis-associated hepatocellular carcinoma development. Int. J. Cancer 136, 2469-2475. doi: $10.1002 /$ ijc. 29287

van Zijl, F., Zulehner, G., Petz, M., Schneller, D., Kornauth, C., Hau, M., et al. (2009). Epithelial-mesenchymal transition in hepatocellular carcinoma. Future Oncol. 5, 1169-1179. doi: 10.2217/fon.09.91
Vincentz, J. W., McWhirter, J. R., Murre, C., Baldini, A., and Furuta, Y. (2005) Fgf15 is required for proper morphogenesis of the mouse cardiac outflow tract. Genesis 41, 192-201. doi: 10.1002/gene.20114

Wang, J., Rhee, S., Palaria, A., and Tremblay, K. D. (2015). FGF signaling is required for anterior but not posterior specification of the murine liver bud. Dev. Dyn. 244, 431-443. doi: 10.1002/dvdy.24215

$\mathrm{Xu}$, J., Lloyd, D. J., Hale, C., Stanislaus, S., Chen, M., Sivits, G., et al. (2009). Fibroblast growth factor 21 reverses hepatic steatosis, increases energy expenditure, and improves insulin sensitivity in diet-induced obese mice. Diabetes 58, 250-259. doi: 10.2337/db08-0392

Yang, H., Fang, F., Chang, R., and Yang, L. (2013). MicroRNA-140-5p suppresses tumor growth and metastasis by targeting transforming growth factor $\beta$ receptor 1 and fibroblast growth factor 9 in hepatocellular carcinoma. Hepatology 58, 205-217. doi: 10.1002/hep.26315

Yilmaz, Y., and Eren, F. (2012). Identification of a support vector machinebased biomarker panel with high sensitivity and specificity for nonalcoholic steatohepatitis. Clin. Chim. 414, 154-157. doi: 10.1016/j.cca.2012.08.005

Zhao, H., Lv, F., Liang, G., Huang, X., Wu, G., Zhang, W., et al. (2015). FGF19 promotes epithelial-mesenchymal transition in hepatocellular carcinoma cells by modulating the GSK3 $\beta / \beta$ - catenin signaling cascade via FGFR4 activation. Oncotarget 7, 13575-13586. doi: 10.1186/1471-2407-12-56

Conflict of Interest Statement: The authors declare that the research was conducted in the absence of any commercial or financial relationships that could be construed as a potential conflict of interest.

Copyright (c) 2016 Itoh, Nakayama and Konishi. This is an open-access article distributed under the terms of the Creative Commons Attribution License (CC BY). The use, distribution or reproduction in other forums is permitted, provided the original author(s) or licensor are credited and that the original publication in this journal is cited, in accordance with accepted academic practice. No use, distribution or reproduction is permitted which does not comply with these terms. 